\title{
Analysis of Laser Phase Noise Effect in Direct- Detection Optical OFDM Transmission
}

\author{
Wei-Ren Peng
}

\begin{abstract}
In this paper, we characterize the impact of laser phase noise (PN) in direct-detection optical OFDM (DDO-OFDM) and identify its many differences from those in coherent optical OFDM (CO-OFDM). The analytical models for the diverse PN effects, including power degradation, phase rotation term (PRT), and inter-carrier interference (ICI), are given in terms of critical system parameters with or without the small PN assumption. In particular, the analytical upper-bound of the ICI power with a simple form is also provided. We also present a bit error rate (BER) estimation approach which is proven to be reliable for an optical signal-to-noise-ratio (OSNR) penalty lower than $\sim 2 \mathrm{~dB}$. In addition, the PN tolerances, in terms of OSNR penalty, are also numerically analyzed and discussed with different QAM formats, data rates, laser linewdiths, and subcarrier numbers.
\end{abstract}

Index Terms-Direct detection, optical fiber communication, orthogonal frequency division multiplexing (OFDM), optical modulation, phase noise.

\section{INTRODUCTION}

$\mathbf{O}$ PTICAL orthogonal-frequency-division-multiplexing (OFDM) has recently received lots of attention due to its high spectral efficiency and superior resilience against fiber chromatic dispersion (CD) [1]-[4]. To date, the OFDM systems have included the coherent and direct detection approaches. The direct-detection optical OFDM (DDO-OFDM) [1], which uses a simpler receiver at a price of worse sensitivity when compared with coherent optical OFDM (CO-OFDM) [2]-[4], could be considered as an alternative modulation format for next-generation metropolitan and long-haul terrestrial transmissions.

One claimed benefit of DDO-OFDM systems is the relaxed requirement for the laser linewidth: contrary to the high-cost external cavity laser (ECL) in CO-OFDM, a low-priced distributed feedback (DFB) laser with a MHz-level linewidth, in general, is considerably acceptable in DDO-OFDM because of its better phase coherency between the carrier and sideband [21]. However, as depicted in Fig. 1, due to fiber CD, the carrier and the sideband would gradually walk off with the increased transmission length and eventually loss their phase coherency and result in significant phase noise (PN) in detection. The laser linewidth effect has been firstly identified in [5] with $12.5 \mathrm{Gbps}$

Manuscript received December 18, 2009; revised June 20, 2010; accepted June 25, 2010. Date of publication July 01, 2010; date of current version August 18,2010

The author is with Department of Photonics and Institute of Electro-Optical Engineering, National Chiao Tung University, HsinChu 300, Taiwan (e-mail: pwr.eo92g@nctu.edu.tw).

Color versions of one or more of the figures in this paper are available online at http://ieeexplore.ieee.org.

Digital Object Identifier 10.1109/JLT.2010.2055834

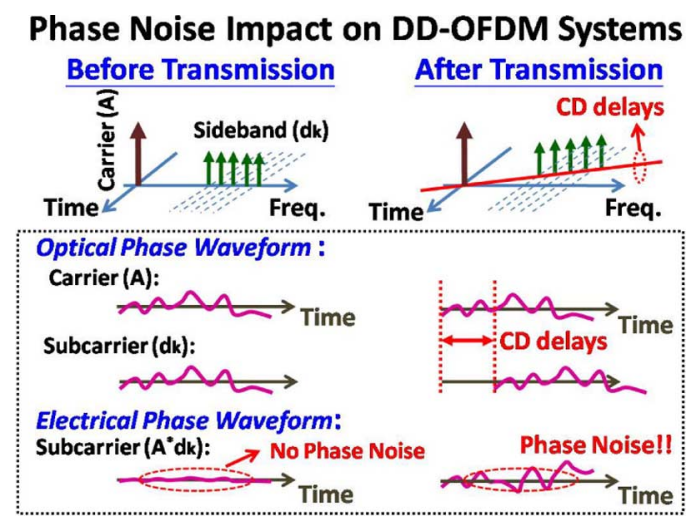

Fig. 1. Origin of phase noise (PN) in DDO-OFDM transmission.

(32-quadrature-amplitude modulation, QAM) data rate and 320-km standard single mode fiber (SSMF) transmission. However, the PN characteristics in DDO-OFDM systems, which are found to be quite different from that in CO-OFDM, should be clarified and characterized. In addition, the PN impact, in terms of its analytical forms with critical system parameters, should be provided from a view point of system design.

In this paper, we firstly present a PN model in DDO-OFDM transmission, characterize and emphasize its many unique properties other than those in CO-OFDM. We find that the PN in DDO-OFDM has the following two significant differences from those in CO-OFDM: 1) the power and bandwidth, i.e., power spectral density (PSD), of PN in DDO-OFDM are functions of the subcarrier frequency and transmission length. Typically, a high-frequency subcarrier or a long-distant transmission would result in a larger PN power and a smaller PN bandwidth. 2) The PN bandwidth could range in $\gg 100 \mathrm{MHz}$ which is independent of the laser linewidth. Such a broad spectrum will inevitably introduce significant inter-carrier interference (ICI), making the PN compensator more difficult to design.

In addition to the PN characterization, we also analyze the PN effects on the received symbol, derive the PN power in terms of the system parameters, and present a simple BER estimator which is proven to be reliable provided the OSNR penalty is smaller than $\sim 2 \mathrm{~dB}$. Furthermore, the numerical results demonstrate that, 1) with the same data rate, the lower QAM format would exhibit a better PN tolerance even though it suffers more $\mathrm{CD}$ and more PN power, 2) the PN tolerance, in terms of the maximum achievable fiber distance, could be easily scaled to different data rates and laser linewidths, which could help simplify the system design, and 3) the PN tolerance could be enhanced by increasing the subcarrier number, which indicates 
that a longer OFDM symbol duration would yield a better performance in the presence of PN.

The rest of this paper is organized as follows. In Section II, the linear system model in the presence of laser PN is provided. In Section III, we analyze the PN effects, derive the closed-form PN power, and offer a semi-analytical BER estimator evaluating the PN-induced OSNR penalty. In Section IV, numerical results and discussions are given with different data rates, laser linewidths, and subcarrier numbers. Finally, Section V concludes this paper.

Notably this paper will focus on the linear PN effect which is mainly comes from the interaction between the laser PN and the fiber $\mathrm{CD}$, and all the presented results and conclusions are obtained without using any PN compensation technique, which currently for DDO-OFDM is still under explored.

\section{SySTEM MODEL}

Throughout this paper we will consider the typical single polarization DDO-OFDM signal [7]-[9], which inserts a frequency gap with the same width as the data sideband and can be modeled by $s(t)$ [8]

$$
s(t)=e^{j 2 \pi\left(f_{A}\right) t}\left(A+\sum_{k=1}^{N_{d}} d_{k} e^{j 2 \pi\left[\left(k+N_{d}\right) \Delta f\right] t}\right)
$$

where $j$ is the imaginary unit, $A$ and $d_{k}$ are the complex amplitudes of the carrier and the modulated symbol on $k$ th subcarrier, respectively, $N_{d}$ is the number of data subcarriers, $f_{A}$ is the carrier's baseband frequency, and $\Delta f$ is the subcarrier frequency spacing. We emphasize that the signal model (1) is compatible with the following DDO-OFDM transmitters with each having different carrier frequency: i) optical-filtering transmitter $\left(f_{A}=0\right)$ [7], which retains the laser carrier and removes one of the sidebands with an optical filter. ii) Digital-carrier transmitter $\left(f_{A}=-N_{d} \Delta f\right)$ [8], which suppresses the laser carrier and inserts a digital carrier by virtual of the digital signal processor (DSP). iii) Analog-carrier transmitter $\left(f_{A}=-1.5 N_{d} \Delta f\right)$ [9], which suppresses the laser carrier and inserts an analog carrier with an external radio frequency (RF) local oscillator. Since the carrier's baseband frequency $f_{A}$ will not take any effect in deriving the $\mathrm{PN}$ model, for simplicity we set its value to be zero, i.e., $f_{A}=0$, for the following analysis.

The phase fluctuation of a laser $\Phi(t)$, which is often quantified by the laser linewidth $\gamma$, can generally be modeled by the Wiener process [6], [10], [12], [15], i.e.,

$$
\Phi(t)=2 \pi \int_{0}^{t} n(v) d v
$$

where $n(v)$ is a zero-mean stationary white Gaussian random process with a variance of $\gamma /(2 \pi)$. As a result, the phase fluctuation $\Phi(t)$ is a normal process with a zero mean and a variance of $\mathrm{t} \gamma /(2 \pi)$. To introduce this phase fluctuation to the typical DDO-OFDM signal (1), we simply multiply (1) with the noisy carrier $\exp (j \Phi(t))$, which by definition is the laser PN. Thus, the signal model with PN can be given as

$$
s_{p}(t)=\left(A+\sum_{k=1}^{N_{d}} d_{k} e^{j 2 \pi\left[\left(k+N_{d}\right) \Delta f\right] t}\right) e^{j \Phi(t)}
$$

For simplicity in (3) we leave out the pulse waveform term, which is typically a rectangular function with a duration defining the period of one OFDM symbol. From the viewpoint of fast Fourier transform (FFT), which presumes that each OFDM symbol extends periodically in the time domain, the above assumption would be still reasonable in the absence of the inter-symbol interference (ISI).

Based on the transmitter's output signal $s_{p}(t)$, because of the power-detection nature of the photodiode, the laser PN will not appear in the converted electrical signal and thus will do no harm to the system performance. However, if after transmission, the signal with a certain amount of $\mathrm{CD}$ will have the different subcarriers experiencing different timing offset, which can be expressed as $s_{t}(t)$

$$
\begin{aligned}
s_{t}(t)= & s_{p}(t) * h(t) \\
= & \left\{\left(A+\sum_{k=1}^{N_{d}} d_{k} e^{j 2 \pi\left[\left(k+N_{d}\right) \Delta f\right] t}\right) e^{j \Phi(t)}\right\} \\
& * \delta\left(t-T_{k}\right) \\
\approx & \left(A e^{j \Phi(t)}+\sum_{k=1}^{N_{d}} d_{k}\right. \\
& \left.\times e^{j 2 \pi\left[\left(k+N_{d}\right) \Delta f\right]\left(t-T_{k}\right)+j \Phi\left(t-T_{k}\right)}\right)
\end{aligned}
$$

where * denotes the convolution operator, $h(t)=\delta\left(t-T_{k}\right)$ is the impulse response of fiber CD with $T_{k}=\left[D L \lambda^{2}(k+\right.$ $\left.\left.N_{d}\right) \Delta f / \mathrm{c}\right]$, of which $D$ is the dispersion parameter, $L$ is the fiber length, $\lambda$ is the operating wavelength, and $c$ is the light speed in vacuum. In deriving (4) we assume that the laser linewidth $\gamma$ is relatively smaller than the subcarrier frequency spacing $\Delta f$ and ignore its finite bandwidth effect. Thus, the fiber CD only contributes a relative time delay $T_{k}$ between the $k$ th subcarrier and the optical carrier.

After the photodiode, the converted electrical signal $R(t)$ will include the desired signal, the direct current (DC), and the sideband-sideband beat interference (SSBI) [8], which are expressed in sequence as follows:

$$
\begin{aligned}
& R(t)=\left|s_{t}(t)\right|^{2} \\
& \approx\left(\begin{array}{rl}
2 \operatorname{Re} & {\left[A^{*} \sum_{k=1}^{N_{d}} d_{k} e^{j 2 \pi\left[\left(k+N_{d}\right) \Delta f\right] t+j \rho_{k}(t)+j \theta_{k}}\right]} \\
+ & {\left[|A|^{2}+\sum_{k=1}^{N_{d}}\left|d_{k}\right|^{2}\right]+\mathrm{SSBI}}
\end{array}\right)
\end{aligned}
$$

where $\rho_{k}(t)=\left[\Phi\left(t-T_{k}\right)-\Phi(t)\right]$ is the converted phase fluctuation on $k$ th subcarrier and therefore $\exp \left(j \rho_{k}(t)\right)$ stands for the converted PN (CPN) on $k$ th subcarrier, $\theta_{k}=(-2 \pi(k+$ 
$\left.N_{d}\right) \Delta f T_{k}$ ) is the CD-induced phase rotation on $k$ th subcarrier, $R e[x]$ takes the real part of $x$, and the superscript ' "*' is the complex conjugator. Before the signal is sampled to the digital domain, we should firstly discuss the PSD of the CPN: $\exp \left[j \rho_{k}(t)\right]$, which is found to be quite different from that of the coherent PN: $\exp [j \Phi(t)]$. The PSD of CPN, denoted as $P_{k}(f)$, had been previously derived in [10] and for convenience we have rewritten it with the herein presented parameters

$$
\begin{aligned}
P_{k}(f)= & e^{-\beta_{k}}\left[\delta(f)-\frac{\sin \left(2 \pi f T_{k}\right)}{\pi f}\right] \\
& +\frac{1}{\pi \gamma\left(1+\left(\frac{f}{\gamma}\right)^{2}\right)}\left\{1-e^{-\beta_{k}}\left[\cos \left(2 \pi f T_{k}\right)\right.\right. \\
& \left.\left.-\frac{f}{\gamma} \sin \left(2 \pi f T_{k}\right)\right]\right\}
\end{aligned}
$$

where $\beta_{k}=2 \pi \gamma T_{k}$ is defined as the PN power on $k$ th subcarrier. Contrary to the PN in CO-OFDM systems, the CPN in DDO-OFDM is a function of the subcarrier index $k$ (or subcarrier frequency). Independent of the laser linewidth, the bandwidth of CPN is also found to be a function of the time delay $T_{k}$ and might range in the order of $\gg 100 \mathrm{MHz}$, which will not simply pollute the $k$ th subcarrier itself but also corrupt its many adjacent subcarriers. Thus, after the signal is sampled and FFT demodulated, the $k$ th subcarrier symbol $R(k)$ can be represented as

$$
\begin{aligned}
R(k)= & D_{k}\left(\frac{1}{N} \sum_{n=0}^{N-1} e^{j \rho_{k}(n)}\right) \\
& +\sum_{m=1, m \neq k}^{N_{d}} D_{m}\left(\frac{1}{N} \sum_{n=0}^{N-1} e^{j\left[\frac{2 \pi(k-m) n}{N}+\rho_{m}(n)\right]}\right) \\
= & D_{k} \psi_{k}(0)+\sum_{m=1, m \neq k}^{N_{d}} D_{m} \psi_{m}(k-m)
\end{aligned}
$$

where $N$ is the FFT size, $D_{k}=A^{*} d_{k} \exp \left(j \theta_{k}\right)$ is the $k$ th subcarrier symbol in the absence of PN, $\rho_{k}(n)$ is the discrete-time converted phase fluctuation, and $\psi_{k}(p)$ is the FFT of $\rho_{k}(n)$, representing the frequency-dependent interferences from $k$ th subcarrier

$$
\psi_{k}(p)=\frac{1}{N} \sum_{n=0}^{N-1} e^{j\left[\frac{2 \pi p n}{N}+\rho_{k}(n)\right]}
$$

From (7) we found that the received $k$ th subcarrier symbol includes not only the transmitted symbol $D_{k}$, which is corrupted by $\psi_{k}(0)$, but also the ICI terms from adjacent symbols $D_{m}$ where $m \neq k$.

It is worth noting that (7) has a similar form as the conventional PN model in CO-OFDM systems [6], [10], [12], [15], except for the specific natures of CPN: 1) the subcarrier frequency dependency and 2) the much-broader bandwidth. In particular, the variance of the 0 th-order interference $\psi_{k}(0)$, conventionally called the common phase error (CPE) in CO-OFDM systems [6], [11], [12], [14], would vary with the subcarrier frequency and would be no longer "common" to all subcarriers, Hence, for

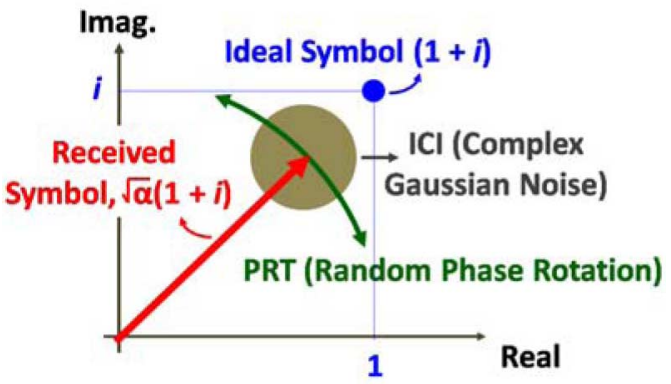

Fig. 2. Effects of the PN on the received subcarrier signal: (1) power degradation $\alpha,(2)$ phase rotation term (PRT), and (3) inter-carrier interference (ICI).

DDO-OFDM we rename $\psi_{k}(0)$ as "phase rotation term (PRT)" to distinguish it from its conventional name of $\mathrm{CPE}$.

\section{Performance AnALYsis}

\section{A. Phase Noise Effects}

The PN effects on the received symbol can be mainly categorized as the power degradation, PRT, and ICI, which can be intuitively understood via Fig. 2. Even though their power, for evaluating the electrical SNR or BER, can be obtained using the numerical approach with the PSD given in (6), we provide their analytical solutions which more clearly identify the direct relations between the PN power and the system parameters.

a) Power Degradation: Since, under the corruption of PN, part of the subcarrier power will leak to the PRT and ICI, the original transmitted subcarrier would inevitably suffer some power loss. The power degradation $\alpha_{k}$, defined as the power ratio between the $k$ th subcarrier with PN to the $k$ th subcarrier without PN can be obtained under the small PN assumption, i.e., $\beta_{k}<0.5$, which in general is true for most practical transmissions. Following the procedures described in Appendix I, $\alpha_{k}$ can be expressed as

$$
\alpha_{k}=\left|E\left\{\psi_{k}(0)\right\}\right|^{2} \approx 1-2 \pi \gamma M_{k} T_{s}=1-\beta_{k}
$$

where $E\{x\}$ takes the expectation of $x, T_{S}$ is the sampling time, and $M_{k}=T_{k} / T_{s}$ is the number of delay samples for $k$ th subcarrier w.r.t the optical carrier. From (9) we found $\alpha_{k}+\beta_{k} \approx 1$, indicating that the power loss of the $k$ th subcarrier will turn to the PN power of $k$ th subcarrier.

b) Phase Rotation Term (PRT): According to the previous definition, PRT is the 0th-order interference $\psi_{k}(0)$. Then, using (8) and the small PN assumption, PRT can be manipulated as follows:

$$
\psi_{k}(0)=\frac{1}{N} \sum_{n=0}^{N-1} e^{j \rho_{k}(n)} \approx 1+j \frac{1}{N} \sum_{n=0}^{N-1} \rho_{k}(n)
$$

We found PRT will only disturb the signal's imaginary part, and therefore results in a phase rotation. Because $\rho_{k}(n)$ is a zero-mean Gaussian random variable, the PRT will also follow Gaussian random process with a zero mean and a variance of

$$
\sigma_{\mathrm{PRT}}^{2} \approx \frac{1}{N^{2}} E\left\{\left(\sum_{n=0}^{N-1} \rho_{k}(n)\right)^{2}\right\}
$$




$$
\begin{aligned}
& =\frac{\sigma^{2} M_{k}}{N^{2}}\left(\frac{-1}{3} M_{k}^{2}+N M_{k}+\frac{1}{3}\right) \\
& =\frac{\beta_{k}}{3 N^{2}}\left(-M_{k}^{2}+3 N M_{k}+1\right)
\end{aligned}
$$

where $\sigma^{2}=2 \pi \gamma T_{s}$ is the $\mathrm{PN}$ variance within a sample duration. The detailed derivation is in Appendix II. Notably in (11) we have not specifically denoted a subscript $k$ for $\sigma_{\mathrm{PRT}}^{2}$; however, the readers should know that the PRT power would always be a function of $k$. In general, because the CP duration is typically longer than the subcarrier' time delay $\left(N \gg M_{k}\right)$, the PRT power (11) could be further simplified as $\sigma_{\mathrm{PRT}}^{2} \approx \beta_{k}\left(M_{k} / N\right)$, which is in proportion to $\left(L^{2} / N\right)$. Therefore, the PRT power will enlarge with an increasing fiber length or/and a decreasing subcarrier number.

c) Inter-Carrier Interference (ICI): The noise behavior of ICI has long been considered as a zero-mean complex Gaussian random variable [6], [12]. The ICI power is also a function of the subcarrier index $k$ and its variance, when normalized to the subcarrier symbol power, can be exactly obtained without using the small PN assumption

$$
\begin{aligned}
\sigma_{\mathrm{ICI}}^{2} & =\frac{E\left\{\left|\sum_{m=1, m \neq k}^{N_{d}} D_{m} \psi_{m}(k-m)\right|^{2}\right\}}{E_{s}} \\
& =\sum_{m=1, m \neq k}^{N_{d}} E\left\{\left|\psi_{m}(k-m)\right|^{2}\right\}
\end{aligned}
$$

with (13), shown at the bottom of the page, where $E_{s}=$ $E\left\{\left|D_{m}\right|^{2}\right\}$ is the subcarrier symbol power in the absence of PN, $d_{1, p}=\exp (j 2 \pi p / N)$, and $d_{2, p}=\exp \left(j 2 \pi p / N-\sigma^{2}\right)$. The exact derivation of (13) is provided in Appendix III. Similar to the PRT power, we also ignore the subscript $k$ for the ICI power denotation while the readers should know that the ICI power will always be a function of $k$. Note that for deriving (12) the following assumption is applied

$$
\begin{aligned}
& E\left\{\left[D_{m 1} \psi_{m 1}(k-m 1)\right]\left[D_{m 2} \psi_{m 2}(k-m 2)\right]^{*}\right\} \\
& \quad=E\left\{D_{m 1} D_{m 2}^{*}\right\} E\left\{\psi_{m 1}(k-m 1) \psi_{m 2}^{*}(k-m 2)\right\} \\
& \quad=0
\end{aligned}
$$

which is true as long as the subcarrier symbols are mutuallyindependent, i.e., $E\left\{D_{m 1} D_{m 2}^{*}\right\}=0$ where $m 1 \neq m 2$.
To simplify (12) and (13), we ignore the PSD difference of CPN for adjacent subcarriers, i.e., $E\left\{\left|\psi_{i}(k-i)\right|^{2}\right\} \approx$ $E\left\{\left|\psi_{k}(k-i)\right|^{2}\right\}$. Therefore, (12) can be approximated as

$$
\begin{aligned}
\sigma_{\mathrm{ICI}}^{2} & \approx \sum_{i=1, i \neq k}^{N_{d}} E\left\{\left|\psi_{k}(k-i)\right|^{2}\right\} \\
& \leq \sum_{i=1, i \neq k}^{N} E\left\{\left|\psi_{k}(i)\right|^{2}\right\}=1-E\left\{\left|\psi_{k}(0)\right|^{2}\right\}
\end{aligned}
$$

where the upper-bound is achieved when $N_{d}=N$. However, if the sideband bandwidth $N_{d} \Delta f$ is larger than the CPN's bandwidth, as is usual the case after a long-distant transmission, the ICI power would mostly come from the adjacent $N_{d}$ subcarriers and thus its value would be very close to this bound.

If we further use the small PN assumption for (15), with which $E\left\{\left|\psi_{k}(0)\right|^{2}\right\}$ can be simply approximated as the sum of the power degradation and PRT power, i.e., $E\left\{\left|\psi_{k}(0)\right|^{2}\right\} \approx$ $\alpha_{k}+\sigma_{\mathrm{PRT}}^{2}$, then the bound in (15) can be re-expressed as

$$
\begin{aligned}
\sigma_{\mathrm{ICI}}^{2} & \leq 1-\left(\alpha_{k}+\sigma_{\mathrm{PRT}}^{2}\right) \\
& =\frac{\beta_{k}}{N^{2}}\left(N^{2}-N M_{k}+\frac{1}{3} M_{k}^{2}-\frac{1}{3}\right)
\end{aligned}
$$

which provides a very simple form for the upper-bound of the ICI power.

\section{B. Bit Error Rate Estimation}

In general, if the PN interferences of PRT and ICI, as well as the ASE beat noise, are mutually independent and follow the complex Gaussian distribution, then the BER can be simply estimated via (12) and (13) in [13]. However, since the PRT will only rotate the subcarrier symbol phase and may not be simply assumed as complex Gaussian noise, an alternate BER estimator would be needed. For deriving the BER estimator, we mainly follow the procedures in [11] and assume that the PRT and ICI are mutually independent so that the conditional BER of $k$ th subcarrier, given a PRT-induced phase rotation $\theta$, can be written as:

$$
\begin{aligned}
\operatorname{BER}_{4}(k, \theta) \approx \frac{1}{4} \operatorname{erfc} & \left(\sqrt{\operatorname{ESNR}_{k}} \cos \left[\frac{\pi}{4}+\theta\right]\right) \\
+ & \frac{1}{4} \operatorname{erfc}\left(\sqrt{\mathrm{ESNR}_{k}} \sin \left[\frac{\pi}{4}+\theta\right]\right)
\end{aligned}
$$

with

$$
\operatorname{ESNR}_{k}=\frac{\alpha_{k}}{\sigma_{\mathrm{ICI}}^{2}+\frac{1}{\operatorname{ESNR}_{\mathrm{ASE}, k}}}
$$

$$
\begin{aligned}
E\left\{\left|\psi_{k}(p)\right|^{2}\right\} & \\
= & \frac{2 e^{-\beta_{k}}}{N^{2}}\left(\operatorname{Re}\left[\frac{d_{1, p}^{N+1}-\left(N-M_{k}+1\right) d_{1, p}^{M_{k}+1}+\left(N-M_{k}\right) d_{1, p}^{M_{k}}}{\left(d_{1, p}-1\right)^{2}}\right]\right) \\
& +\frac{2}{N^{2}}\left(\operatorname{Re}\left[\frac{\left(N-M_{k}+1\right) d_{2, p}^{M_{k}+1}-\left(N-M_{k}\right) d_{2, p}^{M_{k}}-(N+1) d_{2, p}+N}{\left(d_{2, p}-1\right)^{2}}\right]\right)-\frac{1}{N}
\end{aligned}
$$


where the subscript of $\mathrm{BER}_{4}$ stands for the QAM size, i.e., 4 QAM, $\operatorname{erfc}(x)$ is the complement error function, $\operatorname{ESNR}_{k}$ is the $k$ th-subcarrier's electrical signal-to-noise-ratio (ESNR) excluding the PRT power, and $\mathrm{ESNR}_{\mathrm{ASE}, k}$ represents the $k$ th-subcarrier's ESNR with the consideration of only ASE. The numerical method for $\mathrm{ESNR}_{\mathrm{ASE}, k}$ has been well documented in [13] and thus is omitted here. Thus, the ensemble BER of $k$ th subcarrier, with the consideration of all possible PRT phase rotation, can be expressed as [11]

$$
\begin{aligned}
& \mathrm{BER}_{4}(k) \\
& \approx \frac{1}{4} \int_{-\infty}^{\infty}\left(\begin{array}{c}
\operatorname{erfc}\left(\sqrt{\mathrm{ESNR}_{k}} \cos \left[\frac{\pi}{4}+\theta\right]\right) \\
+\operatorname{erfc}\left(\sqrt{\mathrm{ESNR}_{k}} \sin \left[\frac{\pi}{4}+\theta\right]\right)
\end{array}\right) \\
& \quad \times f_{k}(\theta) d \theta
\end{aligned}
$$

where $f_{k}(\theta)$ is the probability density function (PDF) of PRT, which follows the Gaussian distribution with a zero-mean and a variance of $\sigma_{\mathrm{PRT}}^{2}$

$$
f_{k}(\theta)=\frac{1}{\sqrt{2 \pi \sigma_{\mathrm{PRT}}^{2}}} e^{-\frac{\theta^{2}}{2 \sigma_{\mathrm{PRT}}^{2}}}
$$

The system BER can then be obtained by taking the average of $\mathrm{BER}_{4}(k)$ over all subcarriers [13]. Notably the BER with higher QAM formats can be easily derived with a similar form of (26) in [15].

It is worth noting that, given a fixed PN power $\beta_{k}$ (which implies that the total power of PRT and ICI is fixed), because the phase rotation would have a greater impact on BER, the system performance would be worse if the relative power of PRT is higher than that of ICI.

\section{NUMERICAL RESUlTS AND DisCUSSIONS}

Unless specified otherwise, the system parameters used in simulations are given as follows: 16-QAM format, 20-Gbps data rate, $20 \%$ cyclic prefix $(\mathrm{CP}), 160$ data subcarriers $\left(\mathrm{N}_{d}\right), 256 \mathrm{FFT}$ size $(N)$, 2-MHz laser linewidth, and $16 \mathrm{ps} /(\mathrm{nm} . \mathrm{km})$ dispersion parameter. $(1024 \Delta f)$ is chosen as the simulation bandwidth so that the broadband PN impact can be accurately evaluated. The fiber channel used in simulations considers only the linear chromatic dispersion and at the receiver a 2nd-order Gaussian optical filter is applied with a $-3-\mathrm{dB}$ bandwidth equal to $1.2 \times$ $\left(2 N_{d} \Delta f\right)$. No optical dispersion management is deployed in the link, and OSNR is evaluated in a noise bandwidth of $0.1 \mathrm{~nm}$ whenever ASE is considered.

\section{A. Phase Noise Characteristics}

To emphasize the uniqueness of PN in DDO-OFDM systems, in Figs. 3 and 4 we compare the PN characteristics of the COand DDO-OFDM systems. For Figs. 3(a) and 4 we use Monte Carlo (MC) simulations while for Fig. 3(b) we use the analytical PSD given in (6). The ASE noise is ignored to focus on the PN behaviors. In Fig. 3(a) we show the phase fluctuations of 10 consecutive OFDM symbols for the CO- and DDO-OFDM systems with a fiber length of $1600 \mathrm{~km}$. For CO-OFDM we only draw the results of the central subcarrier since the phase fluctuation

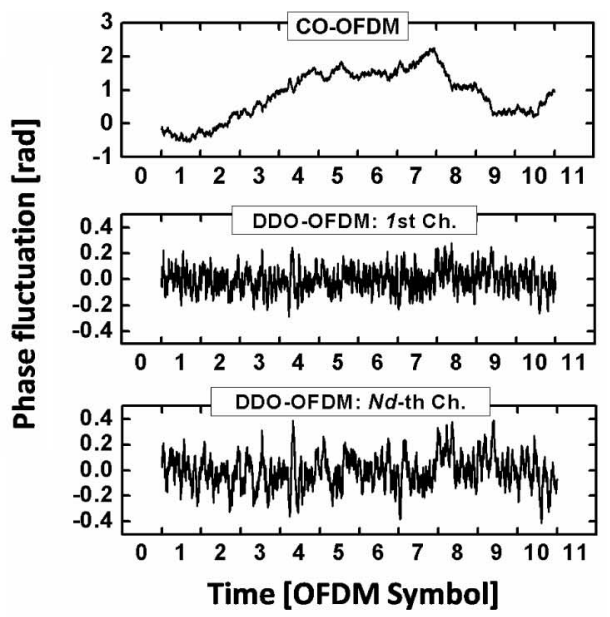

(b)

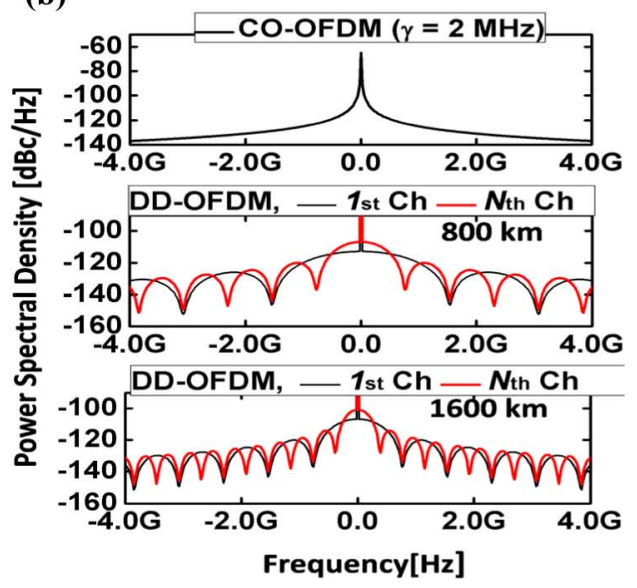

Fig. 3. (a) (Converted) Phase fluctuations versus OFDM symbols for CO-and DDO-OFDM systems. (b) Power spectral density (PSD) of PN. Top: CO-OFDM, Center: DDO-OFDM, with 800-km SSMF, Bottom: DDO-OFDM, with 1600-km SSMF. For all cases the ASE noise is not considered.

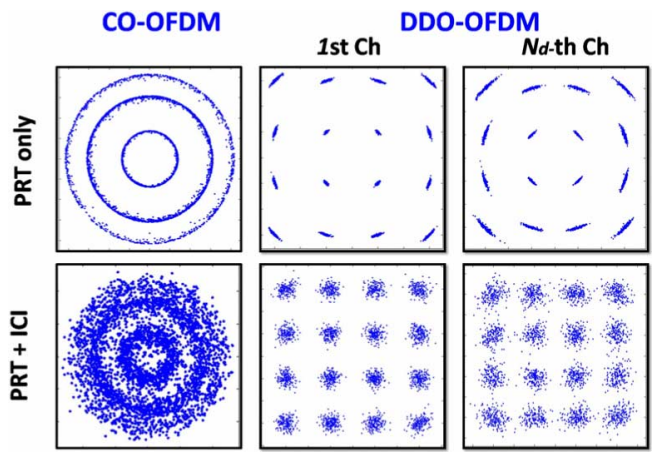

Fig. 4. The effects of phase rotation term (PRT) and ICI on 16-QAM format in both CO- and DDO-OFDM systems. $\gamma=2 \mathrm{MHz} ., L=1600 \mathrm{~km}$. No ASE noise is considered.

will behave similarly among all subcarriers ${ }^{1}$; while for DDO-OFDM we show both the results of 1 st and $\left(N_{d}\right)$-th subcarrier to identify its subcarrier-dependent phase fluctuation characteristic. In CO-OFDM, the larger phase variance

${ }^{1}$ In CO-OFDM, due to the fiber $\mathrm{CD}$, there will also be some relative time delay among the transmitted subcarriers. However, because of the independency between the transmitter laser and the local oscillator, the overall PNs of each subcarrier will behave correlatedly with the same PSD following the Lorenzian shape. 
can be observed because of the random walk nature of the Wiener process, which allows the phase travels unlimitedly with increasing time. On the other hand, the phase variance of DDO-OFDM will be constrained by $\beta_{k}=\left(\sim 2 \pi \gamma T_{k}\right)$ and thus, unlike that in CO-OFDM, the phase will not go unboundedly. Because in DDO-OFDM system the frequency spacing of between the carrier and the $\left(N_{d}\right)$-th subcarrier is $\sim$ doubled than that of between the carrier and the 1st subcarrier, the experienced time delay of $\left(N_{d}\right)$-th subcarrier will also be doubled than that of 1 st subcarrier, i.e., $T_{N d}=2 T_{1}$, thus resulting in an $\sim$ doubled PN power, $\beta_{N d}=2 \beta_{1}$.

Fig. 3(b) shows the PSD of PN in CO-OFDM and DDO-OFDM systems. The PSD of PN in CO-OFDM is essentially follows the Lorentzian shape [10], which has a two-sided 3-dB bandwidth equal to the laser linewidth, i.e., $2 \mathrm{MHz}$ in this demonstration. For DDO-OFDM, we show both the results of 1 st and $\left(N_{d}\right)$-th subcarrier with different fiber lengths of 800-km (center) and 1600-km (bottom). Compared with the PN in CO-OFDM, the PN in DDO-OFDM shows an relatively broader bandwidth (two-sided 3-dB bandwidth = $\sim 350 \mathrm{MHz}$ for 160th subcarrier with $1600 \mathrm{~km}$ transmission), and be functions of both the subcarrier frequency and fiber length. Note that the first frequency null is located at around $\left(1 / T_{k}\right) \mathrm{Hz}^{2}$, which will cover its many adjacent subcarriers as long as the delay time is shorter than the cyclic-prefix (CP), i.e., $T_{k} \leq T_{\mathrm{CP}}<T$, where $T_{\mathrm{CP}}$ is the $\mathrm{CP}$ duration. Such a broad PN spectrum will result in a high ICI power and thus the previously proposed CPE compensator [6], [14] would help limitedly for PN-corrupted DDO-OFDM systems.

In Fig. 4 we show that how the PN will affect the received constellations in both CO-OFDM and DDO-OFDM systems. For CO-OFDM, the PRT (previously CPE) strongly rotates the subcarrier symbol phase so that a digital CPE compensator is a must at the receiver [6]; while for DDO-OFDM, although the PRT also degrade the signal's quality by introducing some phase deviations, the ICI has a more significant impact on the signal's quality. The larger PN power of $\left(N_{d}\right)$-th subcarrier can be clearly observed compared with that of 1 st subcarrier.

Discussions: Contrary to the CO-OFDM, the power and bandwidth of PN in DDO-OFDM are found to be functions of both the subcarrier frequency and transmission distance. Moreover, in a practical link the PN bandwidth might vary around $\gg 100 \mathrm{MHz}$ leading to a significant ICI power. Thus, based on these observations in this section, the previously proposed CPE compensator in CO-OFDM [6], [14], which doesn't take care of the ICI, would inefficiently assist in recovering the PN-corrupted signals in DDO-OFDM ${ }^{3}$. On the other hand, the huge bandwidth of PN in DDO-OFDM would make the previous monitoring-tone compensator [15], [16], which needs an empty frequency band with a width approximately equal to the PN bandwidth, less spectrally-efficient. Therefore, these

\footnotetext{
${ }^{2}$ Based on (2), the converted phase fluctuation $\rho_{k}(t)$ is the integration of the white Gaussian noise $n(t)$ from $(t-T k)$ to $t$, which equivalently filters this while Gaussian noise with a sinc-in-frequency filter with nulls at the multiples of $(1 / T k)$. With the small PN assumption where $\mathrm{CPN} \approx 1+j \rho_{k}(t)$, the PSD of CPN will include the DC carrier and the PSD of $\rho_{k}(t)$, therefore resulting in the spectra in Fig. 3(b).

${ }^{3}$ When the ICI power is much more significant than the PRT power, this scattered-pilot based CPE compensator could even yield a worse performance [20].
}

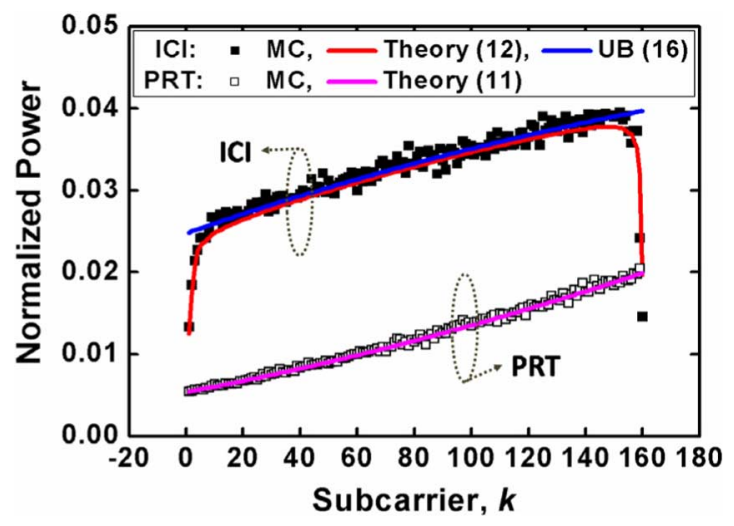

Fig. 5. Power of PRT and ICI for each subcarrier with Monte Carlo (MC) and the analytical approaches. UB: upper bound. The format is 4 QAM and the fiber length is $1500 \mathrm{~km}$.

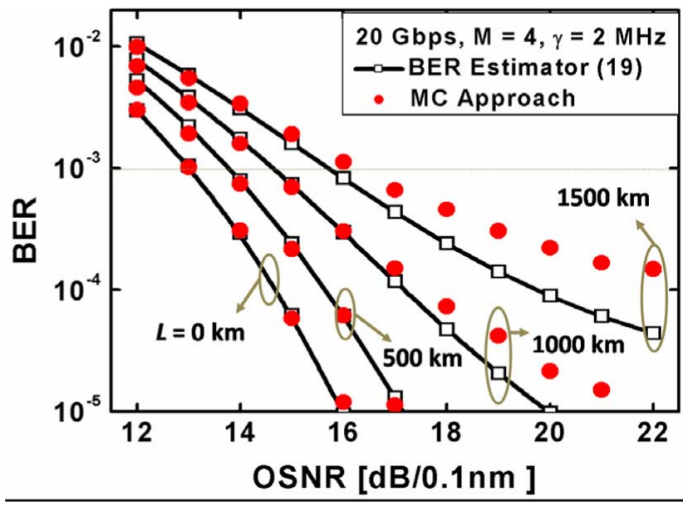

Fig. 6. BER versus OSNR with different transmission distances.

specific characteristics of PN in DDO-OFDM are motivating a new PN compensator for high-capacity transmissions that uses the low-cost DFB lasers.

\section{B. Performance Evaluation}

In this subsection, from Figs. 5 to 8 we will firstly demonstrate the effectiveness of the PN and BER models given in Section III, and then, from Fig. 9 to 11, we show that how the system parameters, such as data rates, laser linewidth, and subcarrier number, will affect the OSNR penalty in the presence of laser PN.

In Fig. 5 we depict the power of PRT and ICI, with both the Monte Carlo (MC) and the presented analytical methods, as a function of subcarrier index in a 4-QAM, 1500-km transmission system. The analytical results, obtained via (11) and (12) for PRT and ICI, respectively, are found to be coincident with the $\mathrm{MC}$ results, thus validating our analytical models for PN. On the other hand, except for the ICI power around the edges, the higher PN power can be found for higher-index (or higher-frequency) subcarriers, which are in agreement with the observations from Figs. 3 and 4. The abrupt drop of the ICI power around the edges can be attributed to their fewer adjacent subcarriers which naturally will contribute less ICI power. In particular, the upper-bound (UB) of the ICI power from (16) is also presented and found to be very close to the exact ICI power for most central subcarriers. Therefore, due to the provided accuracy and its very simple formula, (16) could be directly treated as the ICI power for the future PN-relevant investigations. 
(a)

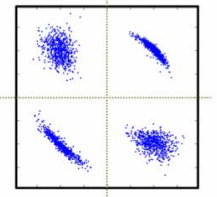

(b)

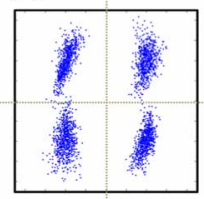

(c)

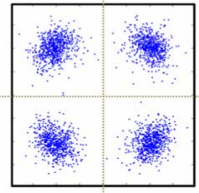

Fig. 7. Constellations of the central (80th) subcarrier signal: (a) with the other 159 subcarriers assigned $(1+i)$, (b) with 1-79 subcarriers assigned $(1+i)$ and with $81-160$ subcarriers assigned $(1-i)$, (c) with all subcarriers assigned random symbols. 4-QAM and 1500-km fiber transmission are considered. The results are obtained using MC approach with no ASE.

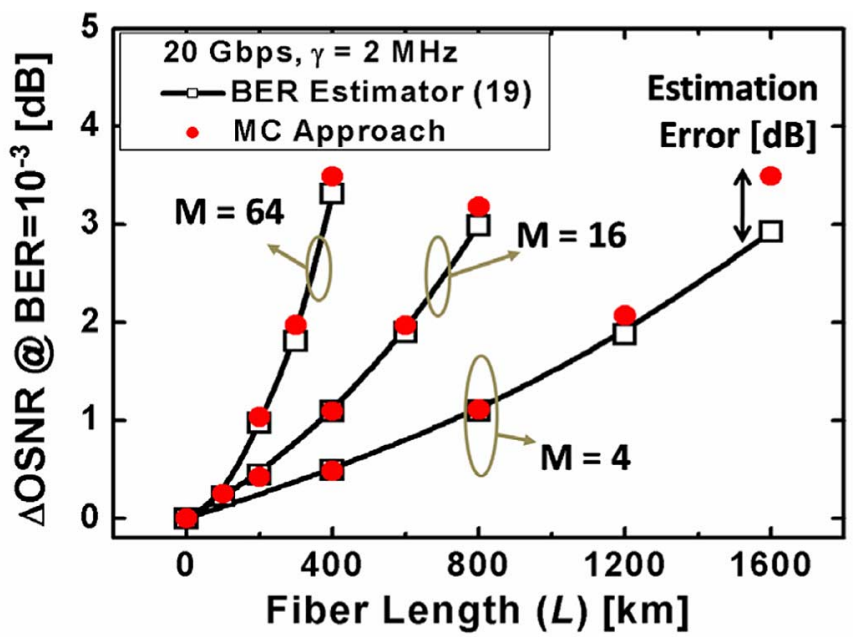

Fig. 8. OSNR penalties versus the fiber length for 4-, 16- and 64-QAMs.

To test the effectiveness of the presented BER estimator, in Fig. 6 we show the BER performances of 4-QAM format as a function of OSNR with both the MC approach and the BER estimator (19). With a shorter fiber length (i.e., $\leq 500 \mathrm{~km}$ ), the results from (19) are found to be well matched to the MC results for a BER down to $10^{-5}$. With a longer distance, however, the estimator becomes unable to yield an accurate BER. Therefore, the BER estimator only can provide reliable results when PN power is relatively smaller than ASE, i.e., under the conditions of either with a lower OSNR or a shorter transmission length. This BER disagreement mainly comes from the data pattern effect of the subcarriers [17], which is not considered for (19).

The pattern effect on the BER estimator can be easily understood via the received constellations. In Fig. 7, the 4-QAM constellations of the 80th subcarrier after $1500-\mathrm{km}$ fiber transmission are shown under different conditions of (a) the rest subcarriers assigned with the symbol of $(1+i)$, (b) the first-half subcarriers (from 1 to 79$)$ assigned with $(1+i)$ and the second halves (from 81 to 160) assigned with $(1-i)$, and (c) the rest subcarriers left random. From Fig. 7(a) and (b) we found that the PN, unlike the regular complex Gaussian noise, scatters in a highly anisotropic manner and exhibits different directivity with different data patterns. However, from Fig. 7(c) the PN would still exhibit the semi-isotropic characteristic since this QAM diagram is assembled from many different data patterns with each contributing its unique directivity. This directly explains why the BER estimator (19) underestimates the exact BER even though the PRT and ICI power, as shown in Fig. 5, can be accu- rately evaluated. Thus, an accurate BER model should take all possible data patterns and the corresponding noise directivity into account. Nevertheless, the computation effort with such an exhausted approach would increase exponentially with the subcarrier number, therefore making such an accurate approach almost prohibitive.

Even though the simple BER model (19) will underestimate the exact BER in the presence of a high PN power, we should discuss the effective range in which (19) could still yield an reliable result. In Fig. 8 we investigate the OSNR penalties versus transmission length with 4-, 16-, and 64-QAM formats. Note that here the different QAMs are switched with a fixed data subcarrier number 160. Both the results from the MC approach and the BER estimator (19) are shown for comparisons. If the maximum estimation error, which is defined as, given a fiber distance $L$, the penalty difference between the $\mathrm{MC}$ results and the estimations by (19), is allowed to be $0.2 \mathrm{~dB}$, we found that (19) can offer an acceptable estimation for both 16- and 64-QAM formats when the OSNR penalty is up to $3 \mathrm{~dB}$; while for the 4-QAM format which suffers stronger PN, (19) can still yield an reliable estimation when the OSNR penalty is $\leq \sim 2 \mathrm{~dB}$. This demonstrates that (19) can reliably estimate the OSNR penalty when the OSNR penalty is $\leq \sim 2 \mathrm{~dB}$ for all the considered QAM formats.

Although in Fig. 8 we have obtained the PN tolerances, in terms of the fiber length, for different QAM formats with a fixed 20-Gbps data rate and a 2-MHz laser linewidth. However, questions would arise whether this PN-tolerance could be scaled to a different data rate or a different laser linewidth. Therefore, in Fig. 9 we discuss the data rate and linewidth scalability of the PN-tolerance in terms of the fiber length. Note that in Figs. 9-11, the results are obtained using MC approach with each point having at least $>100$ bit errors. For simplicity, here we only discuss the 16-QAM format while the other QAMs would yield the similar conclusions. Fig. 9(a) shows the OSNR penalties as a function of fiber length with different data rates $R=10$-, 20and $40 \mathrm{Gbps}$. With a certain OSNR penalty (ex. $3 \mathrm{~dB}$ ), we denote the corresponding fiber length as $L_{R}$ for $R$ data rate. Then the product of $\left(R L_{R}\right)$ is found to be approximately a constant, i.e., $L_{10} \approx 2 L_{20} \approx 4 L_{40}$. Thus, provided the QAM is hold unchanged, the PN-tolerance shown in Fig. 8 could be easily scaled to a different data rate with the rule of $\left(R L_{R}\right) \approx$ constant. Furthermore, in Fig. 9(b) we show the OSNR penalties as a function of fiber length with different linewidths of $\gamma=1,2,4$, and $8 \mathrm{MHz}$. Similarly we define the 3 -dB fiber length to be $L_{\gamma}$, then the product of $\left(\gamma L_{\gamma}\right)$ is found to vary implicitly with different $\gamma$, that is, $L_{1 M} \approx 2 L_{2 M} \approx 4 L_{4 M} \approx 8 L_{8 M}$. Therefore, the PN-tolerance could also be scaled to a different laser linewidth with the relation of $\left(\gamma L_{\gamma}\right) \approx$ constant. The above data rate and linewidth scalability can be clearly understood via the factors of the PN power, $\beta_{k}=2 \pi \gamma T_{k}$. For the same QAM format, the data rate $R$ is roughly in proportion to the subcarrier frequency $f_{k}$, which can be further related to the time delay $T_{k}$ via $T_{k}=C f_{k} L$ where $C$ is a constant. Therefore, given a fixed laser linewidth and a QAM format, a constant $(R L)$ value will result in a constant $\left(f_{k} L\right)$, and thus a constant $T_{k}$ which implies a constant PN power. Therefore, the data-rate scalability simply suggests the 


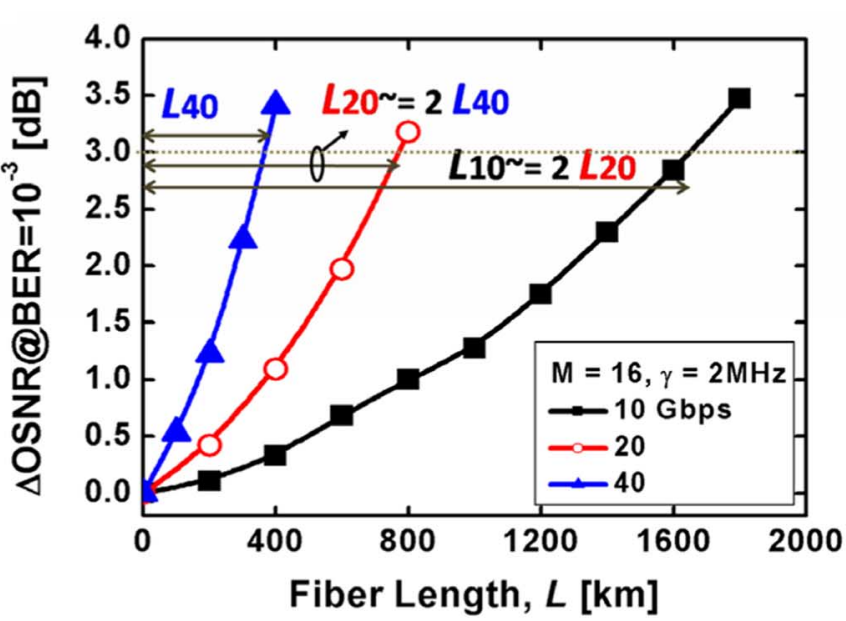

(a)

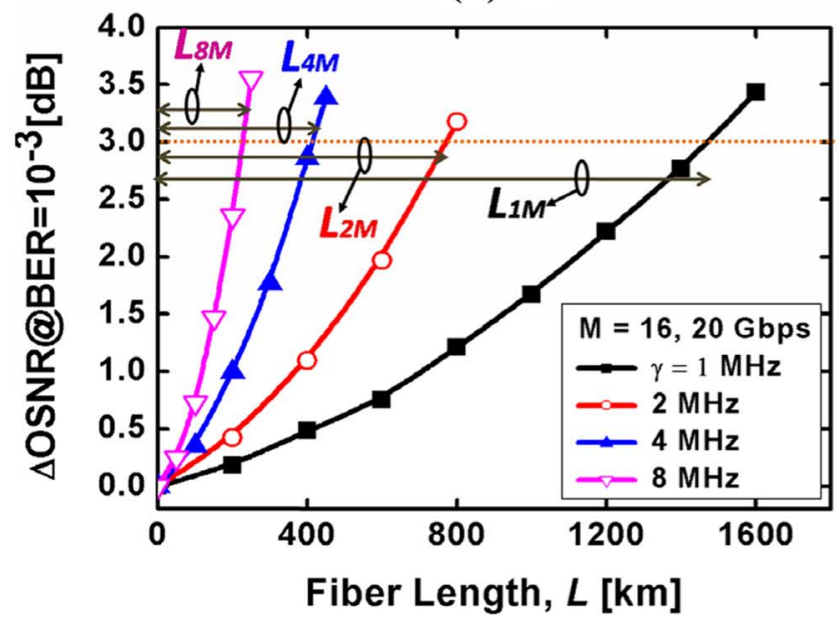

(b)

Fig. 9. OSNR penalties versus fiber length for (a) different data rates, and (b) different laser linewidths.

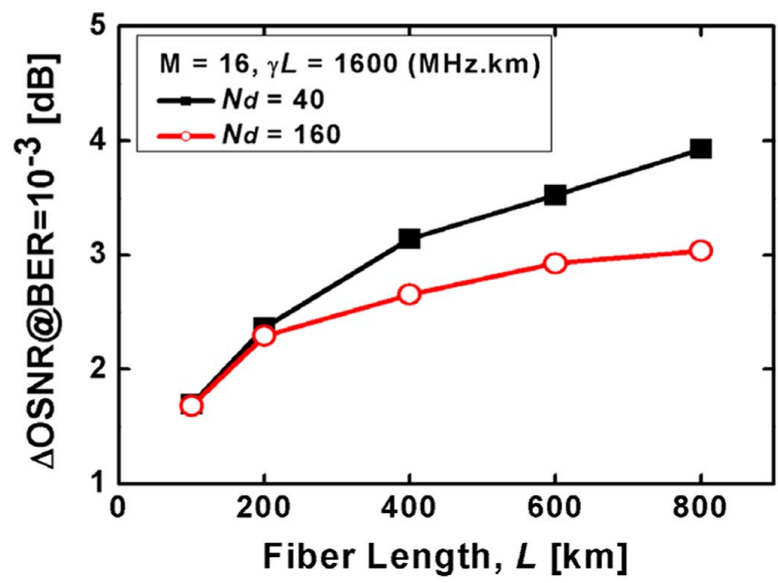

Fig. 10. OSNR penalties versus the fiber length given a fixed $\gamma L$ product of 1600 (MHz.km) yielding a fixed PN power. This emphasizes that the power ratio between PRT and ICI is also important to the OSNR performance.

system would perform similarly provided that the same PN power is given. Likewise, the linewidth scalability can also be realized with a similar manner.

Based on the above discussions, it appears that the OSNR penalty could be easily predicted by the PN power. However, the

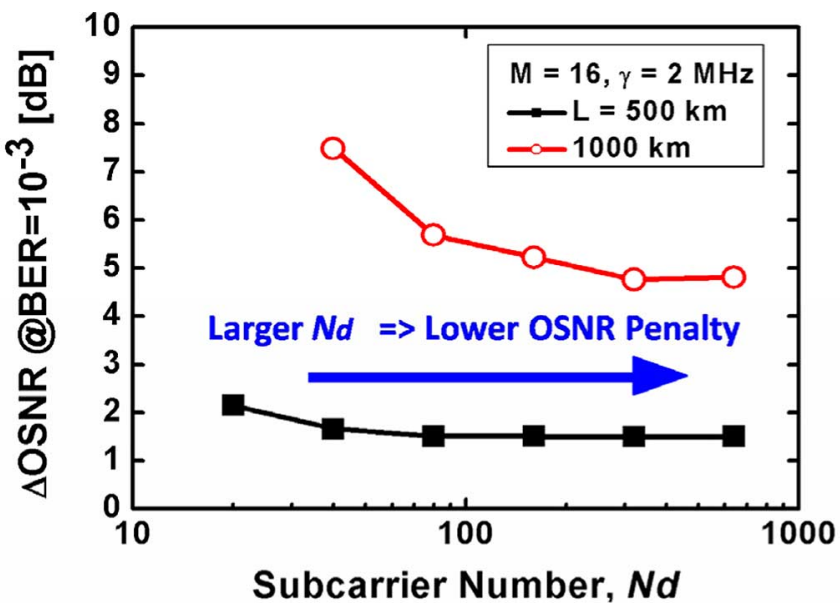

Fig. 11. OSNR penalties versus the subcarrier number with two different transmission lengths. A larger subcarrier number, or alonger symbol duration, is found to have a better performance in the presence of laser PN.

derived data-rate and linewidth scalabilities are simply approximations and the performance would still change even under the same PN power. Shown in Fig. 10 is the OSNR penalty as a function of $L$ given a fixed linewidth and length product of $\gamma L=1600(\mathrm{MHz} \cdot \mathrm{km})$, which yields a fixed PN power. Two different subcarrier numbers $N_{d}=40$ and 160, which correspond to FFT sizes of 64 and 256, respectively, are investigated for comparisons. With both the subcarrier numbers we found the performance would continuously become worse as the length increases, which is more evident especially with the smaller subcarrier number. These observations stems from the following reasons: given a PN power $\left.\beta_{k} \approx \sigma_{\mathrm{PRT}}^{2}+\sigma_{\mathrm{ICI}}^{2}, 1\right)$ a longer distance $L$ would yield a larger $\sigma_{\mathrm{PRT}}^{2}$ through the relation of $\sigma_{\mathrm{PRT}}^{2} \approx \beta_{k}\left(M_{k} / N\right) \propto\left(L^{2} / N\right)$, which indicates that the PRT power would increase with the fiber length and decrease with the subcarrier number. 2) Subsequently, a larger PRT power would lead to a greater OSNR penalty since PRT, rotating randomly the subcarrier symbol phase, typically has a more severe impact than ICI. Thus, even given a fixed PN power, the longer fiber length $L$, or a smaller subcarrier number $\mathrm{N}$, would have a relatively larger OSNR penalty.

Now we investigate how the subcarrier number, i.e., symbol duration, will affect the system performance in the presence of PN. Shown in Fig. 11 are the OSNR penalties versus the subcarrier number with two distinct fiber lengths. Note for Fig. 11 we have removed the constraint of a constant PN power, which is put on Fig. 10. We found 1) the OSNR penalty is more sensitive to the subcarrier number in the case with a longer fiber length, and 2) the greater subcarrier number would yield a lower OSNR penalty for both the considered fiber lengths. In particular, the OSNR improvement brought by the subcarrier number is found to be almost diminished when the subcarrier number is beyond some threshold which depends on the fiber length. As a conclusion for Fig. 11, the longer OFDM symbol duration is more attractive for the better PN tolerance in DDO-OFDM transmissions under the condition of no PN management.

Discussions: Even though the DDO-OFDM signal has long been considered to be immune to fiber $\mathrm{CD}$ and suitable for longdistant transmission, however, this would not be definitely true 
if a low-cost DFB laser is applied in the transmitter. Considering a 16-QAM, 20-Gbps single-band DDO-OFDM transmission [18], a commercial DFB laser with 2-MHz linewidth will limit the 3-dB fiber length to be $<800-\mathrm{km}$, which would constrain the high-capacity DDO-OFDM signals to the local access and metropolitan networks. To increase the PN-limited fiber length, possible strategies might include: 1) Low-QAM format, which, as indicated in this paper, provides a larger PN margin at a price of a lower spectral efficiency. 2) Digital compensators, which, to date, has not been extensively studied for DDO-OFDM. 3) Multi-band transmissions (including the polarization-division multiplexing, PDM), which transmits one carrier for each band to reduce the frequency spacing $f_{k}$ between the carrier and sideband. 4) Virtual SSB-OFDM, which naturally exhibits a higher PN tolerance with the halved signal bandwidth [19]. 5) ECL, which, although with a high price presently, could greatly increase the 3-dB fiber length with its typical linewidth of only $\sim 100 \mathrm{kHz}$. However, among the presented strategies here, if a specified transmission format is given, only (2) and (5) could improve the PN-limited distance, which might lead to a fight between the cost of ECL and the complexity of a digital compensator. Since the aim of this paper is mainly to analyze the PN characteristics and its impact on the system performance, further discussion on the PN compensation is out-of-scope of this paper and should appear in an alternate publication.

\section{CONCLUSION}

We have characterized the PN impact in DDO-OFDM systems and identified its many differences from those in CO-OFDM. The PN effects, categorized as the power degradation, PRT, and ICI, are analyzed in detail and their powers, in terms of critical system parameters, are given with closed-form expressions. Via the numerical results, we have verified the presented PN models and demonstrated that the given BER estimator could be reliable provided that the OSNR penalty is $\leq 2 \mathrm{~dB}$. We have also investigated the PN tolerances, in terms of achievable fiber length given a certain OSNR penalty, with diverse system parameters, such as QAM formats, data rates, laser linewidths, and subcarrier numbers and showed that the PN tolerance could be roughly scaled to a different data rate and laser linewidth which could help simplify the link design process in the future. Finally, for DDO-OFDM a longer OFDM symbol duration is found to have a more robust tolerance against laser PN in the absence of PN management.

\section{APPENDIX I}

For the $k$ th subcarrier, the delayed samples with respect to the optical carrier is denoted as $M_{k}$, that is, $M_{k}=T_{k} / T_{s}$ where
$T_{k}$ is the relative time delay w. r. t. the carrier and $T_{s}$ is the sample duration. Then the discrete-time converted phase fluctuation $\rho_{k}(n)$ can be expressed as the summation of $M_{k}$ mutually-independent Gaussian random variables $v(i)$ starting from $i=\left(n-M_{k}+1\right)$ to $n$

$$
\rho_{k}(n)=\sum_{i=n-M_{k}+1}^{n} v(i)
$$

Note that each single $v(i)$ represents the phase fluctuation within one sample duration with a zero-mean and a variance of $\sigma^{2}=\left(2 \pi \gamma T_{s}\right)$, where $\gamma$ is the laser linewidth.

To derive the power degradation, $\alpha_{k}=\left|E\left\{\psi_{k}(0)\right\}\right|^{2}$ where $E\{x\}$ is the expectation of $x$, we will firstly obtain the expectation of the 0th-order interference, $E\left\{\psi_{k}(0)\right\}$, and then the power degradation will be derived by simply taking the square of it. With small PN assumption, i.e., $e^{x}=1+x+x^{2} / 2, E\left\{\psi_{k}(0)\right\}$ can be manipulated as follows:

$$
\begin{aligned}
E\left\{\psi_{k}(0)\right\} & E\left\{\frac{1}{N} \sum_{n=0}^{N-1} e^{j \sum_{i=n-M_{k}+1}^{n} v(i)}\right\} \\
\simeq & \frac{1}{N} E\left\{\sum _ { n = 0 } ^ { N - 1 } \left(1-\frac{1}{2}\left(\sum_{i=(n+1)-M_{k}}^{n} v(i)\right)^{2}\right.\right. \\
& \left.\left.+j \sum_{i=(n+1)-M_{k}}^{n} v(i)\right)\right\} \\
= & 1-\frac{1}{2 N}\left[\sum_{n=0}^{N-1} E\left\{\left(\sum_{i=(n+1)-M_{k}}^{n} v(i)\right)^{2}\right\}\right] \\
= & 1-\frac{1}{2 N}\left(2 \pi \gamma T_{s} N M_{k}\right)=1-\pi \gamma T_{k}
\end{aligned}
$$

where $E\{v(m) v(n)\}=0$ for $m \neq n$ is used. The power degradation is then obtained via

$$
\alpha_{k}=\left|E\left\{\psi_{k}(0)\right\}\right|^{2} \approx 1-2 \pi \gamma T_{k}=1-\beta_{k} .
$$

\section{APPENDIX II}

The PRT power $\sigma_{\mathrm{PRT}}^{2}$ is the variance of $\psi_{k}(0)$, which can be derived following the below procedures

$$
\sigma_{\mathrm{PRT}}^{2} \approx \frac{1}{N^{2}} E\left\{\left(\sum_{n=0}^{N-1} \rho_{k}(n)\right)^{2}\right\}
$$

$$
\begin{aligned}
E\left\{\left|\psi_{k}(p)\right|^{2}\right\} & =\frac{1}{N^{2}} E\left\{\sum_{n=0}^{N-1} e^{j\left[\frac{2 \pi p n}{N}+\sum_{i=n-M_{k}+1}^{n} v(i)\right]} \sum_{l=0}^{N-1} e^{-j\left[\frac{2 \pi p l}{N}+\sum_{i=l-M_{k}+1}^{l} v(i)\right]}\right\} \\
& =\frac{1}{N^{2}} E\left\{\sum_{n=0}^{N-1} \sum_{l=0}^{N-1} e^{-j\left[\frac{2 \pi p(n-l)}{N}+\sum_{i=l-M_{k}+1}^{l} v(i)-\sum_{i=n-M_{k}+1}^{n} v(i)\right]}\right\}
\end{aligned}
$$




$$
\begin{aligned}
E & \left\{\left|\psi_{k}(p)\right|^{2}\right\} \\
& =\frac{1}{N^{2}} E\left\{\sum_{n=l+M_{k}}^{N-1} \sum_{l=0}^{N-M_{k}-1} e^{j\left[\frac{2 \pi p(n-l)}{N}+\sum_{i=n-M_{k}+1}^{n} v(i)-\sum_{i=l-M_{k}+1}^{l} v(i)\right]}\right\} \\
& =\frac{1}{N^{2}} \sum_{n=l+M_{k}}^{N-1} \sum_{l=0}^{N-M_{k}-1} e^{j \frac{2 \pi p(n-l)}{N}} E\left\{e^{\sum_{i=n-M_{k}+1}^{n} v(i)-\sum_{i=l-M_{k}+1}^{l} v(i)}\right\} \\
& =\frac{1}{N^{2}} \sum_{n=l+M_{k}}^{N-1} \sum_{l=0}^{N-M_{k}-1} e^{j \frac{2 \pi p(n-l)}{N}} e^{-M_{k} \sigma^{2}} \\
& =\frac{e^{-M_{k} \sigma^{2}}}{N^{2}}\left(2 \operatorname{Re}\left[\sum_{h=k}^{N-1} d_{1, p}^{h}(N-h)\right]\right) \\
& =\frac{e^{-M_{k} \sigma^{2}}}{N^{2}}\left(2 \operatorname{Re}\left[\frac{d_{1, p}^{N+1}-\left(N-M_{k}+1\right) d_{1, p}^{M_{k}+1}+\left(N-M_{k}\right) d_{1, p}^{M_{k}}}{\left(1-d_{1, p}\right)^{2}}\right]\right)
\end{aligned}
$$

$$
\begin{aligned}
E\left\{\left|\psi_{k}(p)\right|^{2}\right\} & \frac{1}{N^{2}}\left(2 \Re\left[\sum_{h=0}^{k-1} d_{2, p}^{h}(N-h)\right]-N\right) \\
= & \frac{2}{N^{2}}\left(\Re\left[\frac{\left(N-M_{k}+1\right) d_{2, p}^{M_{k}+1}-\left(N-M_{k}\right) d_{2, p}^{M_{k}}-(N+1) d_{2, p}+N}{\left(d_{2, p}-1\right)^{2}}\right]\right) \\
& -\frac{1}{N}
\end{aligned}
$$

$$
\begin{aligned}
= & \frac{1}{N^{2}} E\left\{\left(\sum_{n=0}^{N-1} \sum_{i=n-M_{k}+1}^{n} v(i)\right)^{2}\right\} \\
= & \frac{1}{N^{2}} E\left\{\mid \sum_{p=1}^{M_{k}-1} p \cdot\left[v\left(-M_{k}+p\right)+v(N-p)\right]\right. \\
& \left.+\left.M_{k} \sum_{q=0}^{N-M_{k}} v(q)\right|^{2}\right\} \\
= & \frac{\sigma^{2}}{N^{2}}\left[2\left(1^{2}+2^{2}+\cdots\left(M_{k}-1\right)^{2}\right)\right. \\
& \left.+\left(N-M_{k}+1\right) M_{k}^{2}\right] \\
= & \frac{\sigma^{2} M_{k}}{N^{2}}\left(\frac{-1}{3} M_{k}^{3}+N M_{k}^{2}+\frac{1}{3} M_{k}\right) .
\end{aligned}
$$

\section{APPENDIX III}

The power of the $p$ th interference bin from $k$ th subcarrier, $E\left\{\left|\psi_{k}(p)\right|^{2}\right\}$, can be expressed as: Depending on the range of $(n-l)$, (A5) will be separately manipulated with the procedures similar to [14]. Here we firstly consider the case for $\mid n-$ $l \mid \geq M_{k}$ : where $R e[x]$ takes the real part of $x$ and $d_{1, p}=$ $\exp (j 2 \pi p / N)$. Similar to the procedures deriving (A6), we can manipulate (A5) for $M_{k}>|n-l| \geq 0$ : where $d_{2, p}=$ $\exp \left(j 2 \pi p / N-\sigma^{2}\right)$.

\section{REFERENCES}

[1] B. J. C. Schmidt, Z. Zan, L. B. Du, and A. J. Lowery, "100 Gbit/s transmission using single-band direct-detection optical OFDM," in Proc. OFC, San Diego, CA, Mar. 2009, Paper PDPC3.

[2] Y. Ma, Q. Yang, Y. Tang, S. Chen, and W. Shieh, "1-Tbps per channel coherent optical OFDM transmission with sub-wavelength bandwidth access," in Proc. OFC, San Diego, CA, Mar. 2009, paper PDPC1.

[3] H. Takahashi, A. Amin, S. L. Jansen, I. Morita, and H. Tanaka, "DWDM transmission with 7.0-bit/s/Hz spectral efficiency using $8 \times 65.1-\mathrm{Gb} / \mathrm{s}$ coherent PDM-OFDM signals," in Proc. Optical Fiber Commun. Conf., San Diego, CA, Mar. 2009, paper PDPB7.

[4] S. L. Jansen, A. Amin, H. Takahashi, I. Morita, and H. Tanaka, "132.2-Gb/s PDM-8QAM-OFDM transmission at 4-b/s/Hz spectral efficiency," IEEE Photon. Technol. Lett., vol. 21, no. 12, pp. 802-804, Jun. 2009.

[5] Z. Zan, M. Premaratne, and A. J. Lowery, "Laser RIN and linewidth requirements for direct detection optical OFDM,' CLEO'08 Paper CWN2.

[6] X. Yi, W. Shieh, and Y. Ma, "Phase noise effects on high spectral efficiency coherent optical OFDM systems," J. Lightw. Technol., vol. 26, no. 10, pp. 1309-1316, May 2008.

[7] A. J. Lowery, L. B. Du, and J. Armstrong, "Performance of optical OFDM in ultra long haul WDM lightwave systems," J. Lightw. Technol., vol. 25, no. 1, pp. 131-138, Jan. 2007, OWM4.

[8] W.-R. Peng, X. Wu, V. R. Arbab, K.-M. Feng, B. Shamee, L. C. Christen, J.-Y. Yang, A. E. Willner, and S. Chi, "Theoretical and experimental investigations of direct-detected RF-tone assisted optical OFDM systems," J. Lightw. Technol., vol. 27, no. 10, pp. 1332-1339, May 2009.

[9] B. J. C. Schmidt, A. J. Lowery, and L. B. Du, "Low sampling rate transmitter for direct-detection optical OFDM system," in Proc. Optical Fiber Commun. Conf., San Diego, CA, Mar. 2009, paper OWM4. 
[10] G. Qi, J. Yao, J. Seregelyi, S. Paquet, C. Belisle, X. Zhang, K. Wu, and R. Kashyap, "Phase-noise analysis of optically generated millimeterwave signals with external optical modulation techniques," J. Lightw. Technol., vol. 24, no. 12, pp. 4861-4875, Dec. 2006.

[11] P. Liu and Y. Bar-Ness, "Closed-form expressions for BER performance in OFDM systems with phase noise," in IEEE Proc. ICC, 2006, pp. 5366-5370.

[12] L. Tomba, "On the effect of Wiener phase noise in OFDM systems," IEEE Trans. Commun., vol. 46, no. 5, pp. 580-583, May 1998.

[13] W.-R. Peng, K.-M. Feng, A. E. Willner, and S. Chi, "Estimation of the bit error rate for direct-detected OFDM signals with optically pre-amplified receivers," J. Lightw. Technol., vol. 27, no. 10, pp. 1340-1346, May 2009.

[14] S. Wu and Y. Bar-Ness, "OFDM systems in the presence of phase noise: Consequences and solutions," IEEE Trans. Commun., vol. 52, no. 11, pp. 1988-1996, Nov. 2004.

[15] M. S. El-Tanany, Y. Wu, and L. Hazy, "Analytical modeling and simulation of phase noise interference in OFDM-based digital television terrestrial broadcasting systems," IEEE Trans. Broadcast., vol. 47, no. 1, pp. 20-31, Mar. 2001

[16] S. L. Jansen, I. Morita, T. C. W. Schenk, N. Takeda, and H. Tanaka, "Coherent optical $25.8 \mathrm{~Gb} / \mathrm{s}$ OFDM transmission over 4160-km SSMF," J. Lightw. Technol., vol. 26, no. 1, pp. 6-15, Jan. 2008.

[17] R. Corvaja and S. Puolin, "Phase noise spectral limits in OFDM systems," Wireless Pers. Commun., vol. 36, pp. 229-244, 2006.

[18] A. Amin, H. Takahashi, I. Morita, and H. Tanaka, "Polarization multiplexed 100-Gbps direct detection OFDM transmission without MIMO processing," in Proc. ECOC'09, Paper 1.3.1.
[19] W.-R. Peng, B. Zhang, K.-M. Feng, X. Wu, A. E. Willner, and S. Chi, "Spectrally efficient direct-detected optical OFDM transmission using a tunable gap and an iterative detection schemes," J. Lightw. Technol., vol. 27, no. 24, pp. 5723-5735, Dec. 2009.

[20] A. G. Armada and M. Calvo, "Phase noise and subcarrier spacing effects on the performance of an OFDM communication system," IEEE Commun. Lett., vol. 2, no. 1, pp. 11-13, Jan. 1998.

[21] D. Qian, J. Yu, J. Hu, L. Zong, L. Xu, and T. Wang, "10 Gb/s WDM-SSB-OFDM transmission over $1000 \mathrm{~km}$ SSMF using conventional DFB lasers and direct-detection," Electron. Lett., vol. 44, no. 3, pp. 223-225, Jan. 2008.

Wei-Ren Peng received the B.S.E.E. degree from National Taiwan University, Taiwan, in 2001, and the M.S. and Ph.D. degrees in electro-optical engineering from National Chiao Tung University, Taiwan, in 2003 and 2008, respectively.

In 2007, he was a visiting researcher at Optical Communications Laboratory at University of Southern California, US, under the supervision of Prof. Alan Willner, working on optical orthogonal frequency division multiplexing (O-OFDM) experiments. In 2009 he worked as a postdoctoral fellow at National Chiao Tung University, Taiwan, focusing on the performance analysis of optical OFDM transmissions. Currently he engages some optical OFDM projects in KDDI R Laboratories, Japan, under contract.

Dr. Peng has authored or coauthored more than 65 papers in prestigious international journals and conferences. He also serves as a paper reviewer for IEEE and OSA journals. His current interest is the impairment compensation using digital signal processing (DSP) for optical long-haul transmissions. 